\title{
Part 2:
}

The role of glutamate, and of excitotoxicity mediated by excess glutamate, in a kaleidoscope of human diseases, and some therapeutic possibilities for silencing glutamate receptors in such conditions 